\title{
Efficacy of Therapeutic Plasma Exchange in Patients with Severe Refractory Anti-NMDA Receptor Encephalitis
}

\author{
Yan Zhang ${ }^{1} \cdot$ Gang Liu $^{1} \cdot$ Mengdi Jiang ${ }^{1} \cdot$ Weibi Chen ${ }^{1} \cdot$ Yingying Su ${ }^{1}$ \\ Published online: 13 March 2019 \\ (C) The American Society for Experimental NeuroTherapeutics, Inc. 2019
}

\begin{abstract}
The objective of the present study was to assess the efficacy of therapeutic plasma exchange (TPE) in patients with severe refractory anti- $N$-methyl-D-aspartate (anti-NMDA) receptor encephalitis. Patients with severe anti-NMDA receptor encephalitis who showed no improvement after steroids and/or intravenous immunoglobulin treatment for at least 10 days were enrolled. All patients received immunotherapy and were divided into a TPE group and a non-TPE group according to treatment received. Each patient in the TPE group received at least 1 TPE course. NMDA receptor antibody titers in the cerebrospinal fluid (CSF) and plasma were evaluated within 1 week after the last TPE procedure. The clinical efficacy of treatment was evaluated after 1 month, 2 months, 3 months, 6 months, and 12 months. Forty patients were enrolled: 19 in the TPE group and 21 in the non-TPE group. Nineteen patients received TPE for a total of 118 procedures. NMDA receptor antibody titers in the CSF and/or plasma decreased or were negative after the last TPE procedure in 18 patients (94.7\%). Compared with the non-TPE group, the TPE group exhibited greater clinical improvement after 1 month and 2 months following treatment $(P<0.05)$. After 3 months, 6 months, and 12 months, there were no significant differences in the outcomes between the TPE group and non-TPE group. The results suggest that TPE might rapidly improve the clinical manifestations in patients with severe refractory anti-NMDA receptor encephalitis, and we believe that TPE should be considered as a first-line treatment.
\end{abstract}

Key Words Anti-N-methyl-D-aspartate receptor encephalitis $\cdot$ therapeutic plasma exchange $\cdot$ severe $\cdot$ refractory $\cdot$ outcome

\section{Introduction}

Anti- $N$-methyl-D-aspartate (anti-NMDA) receptor encephalitis is increasingly recognized as the most common form of immune-mediated encephalitis [1] and has generated considerable attention since it was initially described in 2007 [2]. The majority (75\%) of patients with anti-NMDA receptor encephalitis require extensive critical care management and admission to an intensive care unit (ICU) [3]. The long-term treatment of patients with severe disease is complicated and associated with high medical costs, and treatment for antiNMDA receptor encephalitis is still a big challenge for

Electronic supplementary material The online version of this article (https://doi.org/10.1007/s13311-019-00725-4) contains supplementary material, which is available to authorized users.

Yan Zhang

zhangylq@sina.com

1 Department of Neurology, Xuanwu Hospital, Capital Medical University, Beijing 100053, China neurologists. In the largest cohort study of 577 patients [3], predictors of good outcomes were early treatment and no admission to an ICU. If impairments in autonomic function progress, the disease can be fatal, especially if patients are not adequately treated or are unresponsive to initial treatments.

Common treatment protocols include tumor removal (when present) and first-line immunotherapy, such as steroids, intravenous immunoglobulin (IVIG), and/or therapeutic plasma exchange (TPE) [3, 4]. A direct pathogenic role of antibodies against the GluN1 subunit of the NMDA receptor has been demonstrated in anti-NMDA receptor encephalitis, resulting in immunoglobulin-induced NMDA receptor internalization [5], which supports the rationale for pathogenic antibody removal for treatment. TPE is an established and often life-saving treatment for several conditions [4]. Although preliminary data have suggested that corticosteroids may not be as effective as steroids followed by TPE [6], it still remains unclear whether TPE is superior to steroids and/or IVIG. In addition, there is a paucity of published reports on the role of TPE in the management of severe anti-NMDA 
receptor encephalitis. Because patients with severe disease may have abnormal autonomic nervous system function and hemodynamic instability, whether they can tolerate TPE procedures and the long-term outcomes after TPE remains unclear. Therefore, we conducted this prospective study to explore the efficacy of TPE for the treatment of severe antiNMDA receptor encephalitis in patients who had no response to early steroids and/or IVIG.

\section{Methods}

\section{Trial Registration}

This study was approved by the Ethics Committee of Xuanwu Hospital, Capital Medical University, adhered to the tenets of the Declaration of Helsinki, was registered in the Chinese Clinical Trial Registry (ChiCTR-TRC-14004931), and was conducted in Xuanwu Hospital, Capital Medical University, which is a tertiary care teaching hospital specialized in neurology.

\section{Patient Eligibility}

Patients with anti-NMDA receptor encephalitis who were consecutively admitted to the Department of Neurology in Xuanwu Hospital of Capital Medical University between January 1, 2012, and June 30, 2017, were enrolled. The inclusion criteria were as follows: 1) patients who met the antiNMDA receptor encephalitis diagnostic criteria [7], which include a rapid onset (less than 3 months) of one or more of the six major groups of symptoms (such as abnormal behavior or cognitive dysfunction, speech dysfunction, seizures, movement disorders, decreased level of consciousness, and autonomic dysfunction or central hypoventilation), the presence of IgG anti-GluN1 antibodies, and reasonable exclusion of other disorders; 2) critically ill patients who had a modified Rankin scale (mRS) score $[3,8]$ (Table 1) of 4 to 5 , respiratory failure requiring mechanical ventilation, disturbances of consciousness, or status epilepticus; 3 ) patients who had no improvement after steroid and/or IVIG treatment for at least 10 days; and 4) patients for whom informed consent was obtained from their families.

\section{Treatment Protocol}

All patients received tumor screening, symptomatic supportive treatment, and immunotherapy. All patients with tumors underwent tumor resection. First-line immunotherapy included intravenous glucocorticoid therapy (1000 mg or $500 \mathrm{mg}$ methylprednisolone for 3 or 5 days), IVIG $(0.4 \mathrm{~g} / \mathrm{kg}$ /day for each course for 5 days), and/or TPE (3-5 procedures in each course).
Table 1 Modified Rankin scale

\begin{tabular}{ll}
\hline Level & Description \\
\hline 0 & No symptoms \\
1 & No significant disability, despite symptoms; able \\
to perform all usual duties and activities \\
2 & $\begin{array}{c}\text { Slight disability; unable to perform all previous activities } \\
\text { but able to look after own affairs without assistance }\end{array}$ \\
3 & Moderate disability; requires some help, but able to \\
& walk without assistance \\
4 & Moderately severe disability; unable to walk \\
& without assistance and unable to attend to \\
& own bodily needs without assistance
\end{tabular}

The relative contraindications of TPE included severe active hemorrhage, disseminated intravascular coagulation, severe hypotension or shock, unstable cardiac failure, cerebral hernia, severe infection, severe abnormal mental behaviors, and other dangerous conditions. Patients without the relative contraindications and whose families signed an informed consent form were treated with TPE. TPE treatments were performed using a MultiFiltrate apheresis device (Fresenius, Germany). Each patient received at least 1 TPE course. TPE volumes of 1 plasma volume per procedure were used. Treatments were administered every other day, with breaks allowed for weekends for most patients. Five percent of albumin and plasma was used as a replacement fluid. The patients accepted more courses of TPE if their plasma or cerebrospinal fluid (CSF) NMDA receptor antibody titers did not reduce after 1 course of TPE. Corticosteroids and/or IVIG were administered to patients who did not receive TPE. According to the treatment of TPE, patients were divided into a TPE group and a non-TPE group.

Patients who had no improvement after first-line immunotherapy and whose families signed an informed consent received second-line immunotherapy with immunosuppressants (rituximab, cyclophosphamide, mycophenolate mofetil, or azathioprine).

\section{Extent of Complications and Adverse Events Associated with TPE}

During TPE, electrocardiography, respiration, pulse, blood pressure, and blood oxygen were monitored continuously. Monitoring was also performed for allergic reactions, bleeding, TPE tube coagulation, and filter failure. We rated the complications and adverse events associated with TPE as follows: grade I was defined as mild (no intervention required); grade II was defined as moderate (intervention required but TPE treatment was completed); grade III was defined as 
severe (TPE procedure was interrupted or abandoned); and grade IV was defined as fatal [9].

\section{Data Collection}

The following information was recorded during the study and was obtained from the study data files: age, gender, time of onset, clinical manifestations, presence of teratomas or other tumors, NMDA receptor antibody titers in the CSF and plasma, mRS score upon study enrollment, the number of days between disease onset and immunotherapy, adverse events up to 7 days after TPE, treatment of adverse events, and clinical outcomes.

\section{Efficacy of TPE and Prognostic Evaluation}

In the TPE group, the efficacy was evaluated after each TPE course, including antibody titer and clinical evaluations. NMDA receptor antibody titers in the CSF and plasma were evaluated within 1 week after the last TPE procedure. In the non-TPE group, NMDA receptor antibody titers in the CSF and plasma were evaluated after administering IVIG 23 weeks later. The mRS $[3,8]$ was used to evaluate the clinical efficacy after 1 month, 2 months, and 3 months after enrollment in this study. The improvement of clinical manifestations was defined by an mRS score decrease of at least 1 .

Patients were followed up at 6 and 12 months after enrollment. The mRS $[3,8]$ was used for outcome evaluations. After discharge, outcome evaluations were performed during a clinical visit to an experienced neurologist who was blinded to the clinical manifestations and treatments or performed by telephone follow-up. The evaluation standards were as follows: an mRS score of 0-2 indicated a favorable outcome, and an mRS score of 3-6 indicated an unfavorable outcome.

\section{Statistical Analysis}

Statistical analyses were performed with the statistical software SPSS 22.0 (IBM Corporation, Armonk, NY). Data with a normal distribution are expressed as the means \pm standard deviations. Student's $t$ test was used for intergroup comparisons of data with a normal distribution and homogeneous variance. Binary data were analyzed using Fisher's exact test to examine the differences in each observed indicator between the TPE and non-TPE groups. $P \leq 0.05$ was deemed statistically significant.

\section{Results}

This study enrolled 40 patients with severe refractory antiNMDA receptor encephalitis, including 19 males $(47.5 \%)$ and 21 females $(52.5 \%)$ (Table 2). The ages of the patients were between 12 and 62 (mean, $28.1 \pm 12.6$ ) years. Of the 40 patients, 19 were in the TPE group and 21 were in the nonTPE group. Among the 21 patients in the non-TPE group, 12 did not have their families' informed consent for TPE, 4 had severe hypotension, 3 had a severe infection, and 2 had severe abnormal mental behaviors.

Clinical manifestations and treatments are shown in Table 2. Thirty-three patients had abnormal mental behaviors, including balderdash in 14 , apathy in 8 , hallucination in 7 , and aggressive behaviors in 4 . Six female patients $(6 / 40,15.0 \%)$ had ovarian teratomas, and all underwent teratoma resection within 1 month of admission. Compared with the non-TPE group, the TPE group exhibited higher rates of epilepsy and involuntary movements $(P<0.05)$.

Nineteen patients received TPE for a total of 118 procedures (Table 3). Thirteen patients received 1 TPE course, 5 received 2 TPE courses, and 1 received 3 TPE courses. The majority of patients received an average of 5 TPE procedures (range, 3 to 15 procedures). The number of days from disease onset to initiation of TPE ranged from 12 to 83 days (46 \pm 20 days).

Complications and adverse events associated with TPE are shown in Table 4. During the 118 TPE procedures, the occurrence of involuntary movements increased during 43 (36.4\%) procedures in patients who already had involuntary movements prior to the TPE procedures; during 42 of these 43 procedures, patients needed sedatives or increased doses of the original sedatives to continue TPE. Hypotension occurred during $30(25.4 \%)$ procedures, including transient hypotensive episodes that responded to either a fluid bolus or vasopressor treatment during 29 procedures and more serious hypotension $(65 / 40 \mathrm{mmHg})$ that required discontinuation of TPE during 1 procedure. Clots in the TPE tube occurred during 2 $(1.7 \%)$ procedures, and TPE had to be discontinued. The patient's body temperature increased during $1(0.8 \%)$ procedure, and it returned to normal $4 \mathrm{~h}$ post-TPE. One patient experienced an anaphylactic reaction that manifested as bilateral conjunctival edema during $1(0.8 \%)$ procedure; the patient was treated with anti-allergic therapy, and the symptoms disappeared after 1 day. Statistical analysis of various monitoring indexes before and after each TPE procedure showed that the patients' changes in systolic blood pressure, diastolic blood pressure, and heart rate had no meaningful differences (Fig. 1).

Clinical efficacy and outcomes are shown in Table 2. In the comparison of the improvements in clinical manifestations between the TPE group and the non-TPE group, there were significant differences after 1 month and 2 months following treatment. Compared with the non-TPE group, the TPE group exhibited greater clinical improvement after 1 month and 2 months. In the TPE group, the majority of patients tolerated the TPE procedures well and showed responses in terms of clinical manifestations and NMDA receptor antibody titers in 
Table 2 Demographics, clinical manifestations, treatments, and outcomes of patients with severe anti-NMDA receptor encephalitis

\begin{tabular}{|c|c|c|c|c|}
\hline & Total $(n=40)$ & Non-TPE $(n=21)$ & TPE $(n=19)$ & $P$ value \\
\hline \multicolumn{5}{|l|}{ Gender $(n, \%)$} \\
\hline Male & $19(47.5)$ & $11(52.4)$ & $8(42.1)$ & 0.545 \\
\hline Female & $21(52.5)$ & $10(47.6)$ & $11(57.9)$ & \\
\hline Age (years, mean $\pm \mathrm{SD}$ ) & $28.1 \pm 12.6$ & $30.7 \pm 14.0$ & $25.2 \pm 10.5$ & 0.171 \\
\hline \multicolumn{5}{|l|}{ Tumor comorbidity $(n, \%)$} \\
\hline Ovarian teratoma & $4(15.0)$ & $1(4.8)$ & $5(25.3)$ & 0.085 \\
\hline No tumor & $34(85.0)$ & $20(95.2)$ & $14(73.7)$ & \\
\hline \multicolumn{5}{|l|}{ Clinical manifestations $(n, \%)$} \\
\hline Disturbance of consciousness & $37(92.5)$ & $18(85.7)$ & $19(100)$ & 0.233 \\
\hline Involuntary movements & $35(87.5)$ & $16(76.2)$ & $19(100)$ & 0.049 \\
\hline Mental behavior disorder & $33(82.5)$ & $16(76.2)$ & $17(89.5)$ & 0.412 \\
\hline Epileptic seizures & $31(77.5)$ & $12(57.1)$ & $19(100)$ & 0.001 \\
\hline Status epilepticus & $3(7.5)$ & $2(9.5)$ & $1(5.3)$ & 1.000 \\
\hline Central hypoventilation & $24(60.0)$ & $10(47.6)$ & $14(73.7)$ & 0.117 \\
\hline Cognitive impairment & $22(55.0)$ & $9(42.9)$ & $13(68.4)$ & 0.125 \\
\hline Autonomic nervous dysfunction & $16(40.0)$ & $10(47.6)$ & $6(31.6)$ & 0.349 \\
\hline Days between onset and immunotherapy (mean \pm SD) & $22.3 \pm 11.8$ & $24.3 \pm 10.8$ & $20.0 \pm 12.9$ & 0.258 \\
\hline Hormonotherapy (n, \%) & $37(92.5)$ & $18(85.7)$ & $19(100)$ & 0.233 \\
\hline Days between onset and hormonotherapy (mean $\pm \mathrm{SD}$ ) & $25.3 \pm 16.2$ & $25.3 \pm 15.7$ & $25.3 \pm 17.2$ & 0.990 \\
\hline IVIG $(\mathrm{n}, \%)$ & $40(100)$ & $21(100)$ & $19(100)$ & - \\
\hline Days between onset and IVIG (mean \pm SD) & $25.3 \pm 14.0$ & $27.4 \pm 10.8$ & $22.8 \pm 16.8$ & 0.306 \\
\hline Immunosuppressants $(n, \%)$ & $21(52.5)$ & $10(47.6)$ & $11(37.9)$ & 0.545 \\
\hline RTX & $6(15.0)$ & $1(4.8)$ & $5(26.3)$ & 0.085 \\
\hline CYC & $3(7.5)$ & $1(4.8)$ & $2(10.5)$ & 0.596 \\
\hline MMF & $10(25.0)$ & $6(28.6)$ & $4(21.1)$ & 0.721 \\
\hline AZA & $5(12.5)$ & $2(9.5)$ & $3(15.8)$ & 0.654 \\
\hline Days between onset and immunosuppressants (mean \pm SD) & $61.8 \pm 10.6$ & $57.6 \pm 8.4$ & $65.6 \pm 11.2$ & 0.081 \\
\hline Days of consciousness disorder (mean $\pm \mathrm{SD})$ & $71.1 \pm 36.2$ & $86.5 \pm 30.9$ & $55.6 \pm 35.3$ & 0.010 \\
\hline Days on ventilator supporting (mean $\pm \mathrm{SD}$ ) & $30.3 \pm 23.7$ & $34.9 \pm 33.1$ & $26.7 \pm 13.2$ & 0.422 \\
\hline Duration of involuntary movement (days, mean $\pm \mathrm{SD}$ ) & $26.4 \pm 11.0$ & $28.8 \pm 10.6$ & $24.3 \pm 11.3$ & 0.236 \\
\hline Duration of epileptic seizures (days, mean \pm SD) & $16.4 \pm 7.2$ & $20.6 \pm 6.1$ & $13.7 \pm 6.6$ & 0.007 \\
\hline \multicolumn{5}{|l|}{ mRS score before enrollment $(n, \%)$} \\
\hline 4 & $1(2.5)$ & $0(0)$ & $1(5.3)$ & 0.475 \\
\hline 5 & $39(97.5)$ & $21(100)$ & $18(94.7)$ & \\
\hline \multicolumn{5}{|l|}{ Clinical improvement after 1 month $(n, \%)$} \\
\hline No & $33(82.5)$ & $20(95.2)$ & $13(68.4)$ & 0.040 \\
\hline Yes & $7(17.5)$ & $1(4.8)$ & $6(31.6)$ & \\
\hline \multicolumn{5}{|l|}{ Clinical improvement after 2 months $(n, \%)$} \\
\hline No & $24(60.0)$ & $17(81.0)$ & $7(36.8)$ & 0.009 \\
\hline Yes & $16(40.0)$ & $4(19.0)$ & $12(63.2)$ & \\
\hline \multicolumn{5}{|l|}{ Clinical improvement after 3 months $(n, \%)$} \\
\hline No & $9(22.5)$ & $7(33.3)$ & $2(10.5)$ & 0.133 \\
\hline Yes & $31(77.5)$ & $14(66.7)$ & $17(89.5)$ & \\
\hline \multicolumn{5}{|l|}{ mRS score after 6 months $(n, \%)$} \\
\hline $0-2$ & $10(25.0)$ & $6(28.6)$ & $4(21.1)$ & 0.721 \\
\hline $3-6$ & $30(75.0)$ & $15(71.4)$ & $15(78.9)$ & \\
\hline \multicolumn{5}{|l|}{ mRS score after 12 months $(n, \%)$} \\
\hline $0-2$ & $5(12.5)$ & $3(14.3)$ & $2(10.5)$ & 1.000 \\
\hline $3-6$ & $35(87.5)$ & $18(85.7)$ & $17(89.5)$ & \\
\hline
\end{tabular}

$\mathrm{TPE}=$ therapeutic plasma exchange; $\mathrm{IVIG}=$ intravenous immunoglobulin; $\mathrm{mRS}=$ modified Rankin scale; $\mathrm{RTX}=$ rituximab; $\mathrm{CYC}=$ cyclophosphamide; $\mathrm{MMF}=$ mycophenolate mofetil; $\mathrm{AZA}=$ azathioprine

the CSF and plasma after completion of TPE therapy. In the TPE group, NMDA receptor antibody titers in the CSF and/or plasma decreased or were negative after the last TPE procedure in 18 patients (94.7\%) (Table 3). Among them, the NMDA receptor antibody titers in the CSF decreased after TPE in 12 patients (63.2\%), and plasma titers decreased or 


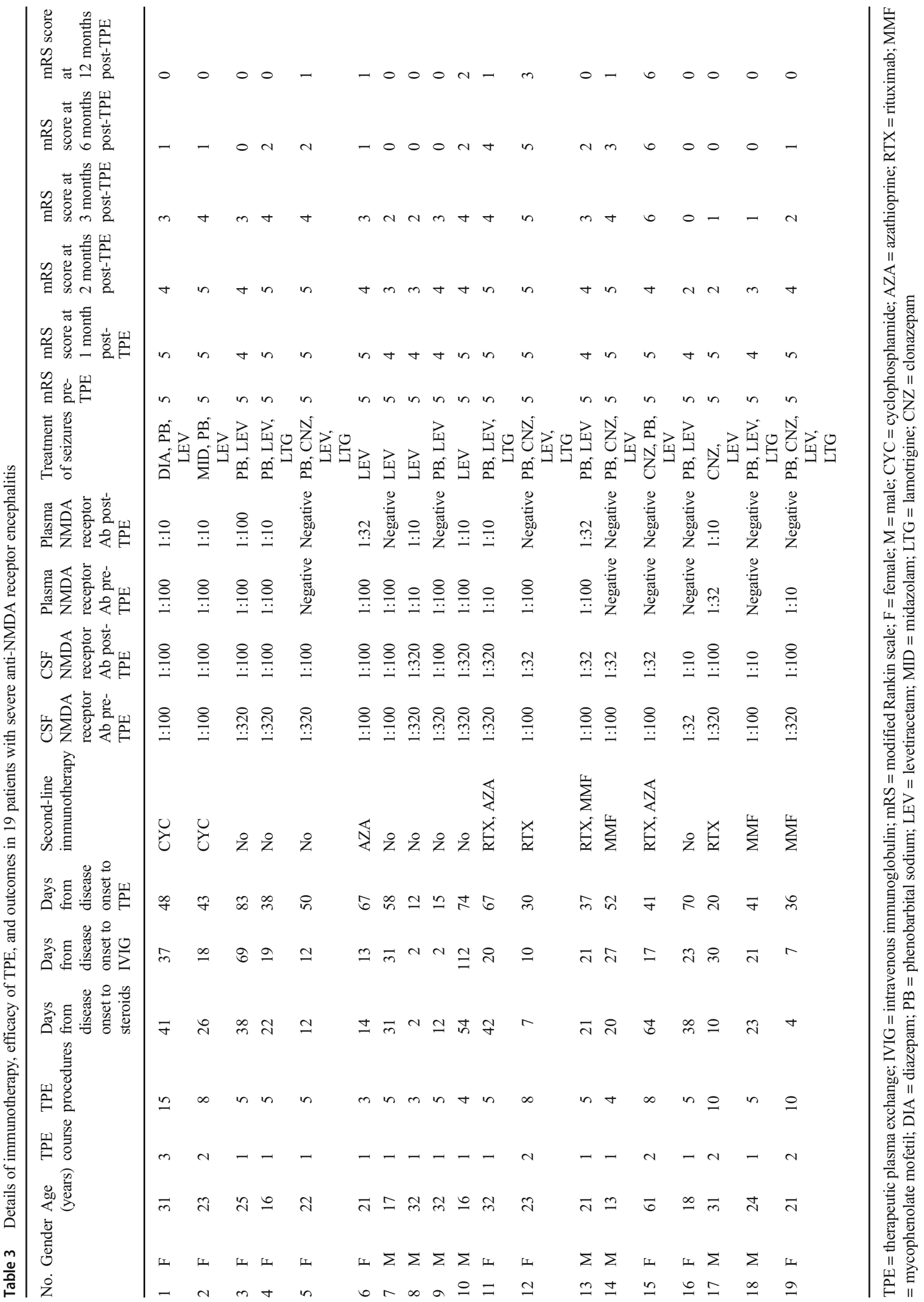


Table 4 Severity of complications and adverse events associated with TPE

\begin{tabular}{llcc}
\hline Grading & Complications and adverse events & Number of events & Percentage of total TPE procedures \\
\hline \multirow{2}{*}{ Grade I } & Fever & 1 & 0.8 \\
& Involuntary movements & 1 & 0.8 \\
\multirow{2}{*}{ Grade II } & Hypotension & 29 & 24.6 \\
& Anaphylactic reaction & 1 & 0.8 \\
& Involuntary movements & 42 & 35.6 \\
Grade III & Hypotension & 1 & 0.8 \\
& Clot in the tube & 2 & 1.7 \\
\hline
\end{tabular}

$\mathrm{TPE}=$ therapeutic plasma exchange were negative after TPE in 13 patients (68.4\%). NMDA receptor antibody titers in the CSF and plasma remained unchanged in a patient who did not receive repeated TPE due to severe hypotension. In the non-TPE group, NMDA receptor antibody titers in the CSF and/or plasma decreased or were negative after administering IVIG in 7 patients (38.9\%) among the 18 patients with recheck results (Table 5). After 3 months, 6 months, and 12 months, there were no significant differences in the outcomes between the TPE group and the non-TPE group. Two patients died in this study: one patient in the TPE group showed marked improvement in neurological function 2 months after TPE but died 1 month after discharge due to liver failure; the other patient in the non-TPE group died of multiple organ failure 1 month after enrollment.

\section{Discussion}

The results of this study confirm that TPE may be rapidly effective in patients with severe refractory anti-NMDA receptor encephalitis. The TPE group had higher rates of epilepsy and involuntary movements than the non-TPE group did, indicating that the patients in the TPE group had more severe encephalitis. Additionally, the TPE group exhibited greater clinical improvement after 1 month and 2 months, although there were no significant differences at 3,6 , or 12 months. Thus, early TPE treatment (as soon as possible and within

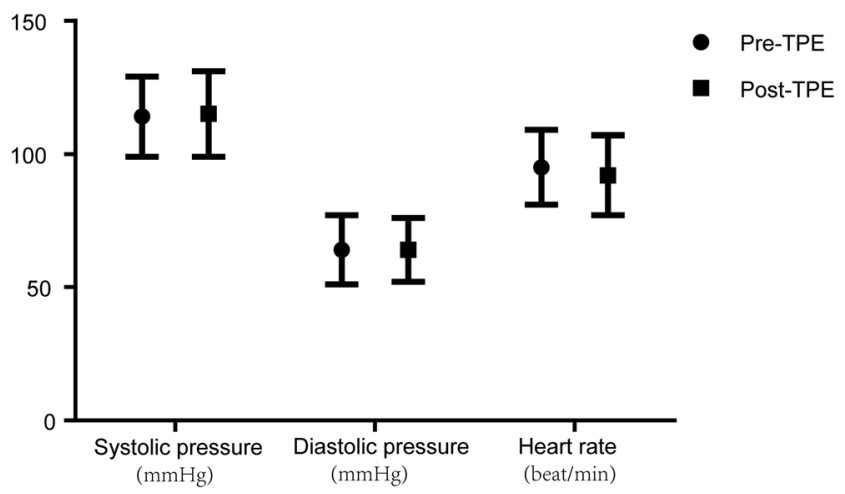

Fig. 1 Systolic blood pressure, diastolic blood pressure, and heart rate before and after each TPE procedure
2 months) is very important for severe patients for whom TPE can relieve symptoms, improve consciousness, and reduce the dosage of sedatives for the treatment of epilepsy. These preliminary data suggest that corticosteroids and/or IVIG may not be as rapidly effective as TPE combined with steroids and IVIG for patients with severe refractory antiNMDA receptor encephalitis. We believe that TPE should be considered as a first-line treatment with steroids before IVIG and should be administered earlier in severe cases.

TPE is a therapeutic apheresis procedure used to remove large molecular substances from the blood and can be used as first-line immunotherapy for anti-NMDA receptor encephalitis $[3,4]$. TPE removes pathogenic antibodies in combination with immunotherapy to suppress antibody production and tumor excision, if present, to remove any possible antibody stimulus. A case series [10] suggested that early initiation of TPE or TPE followed by IVIG led to better outcomes in patients with anti-NMDA receptor encephalitis. Furthermore, fewer patients showed improvement following steroid treatment than the number of patients who improved immediately following TPE $[11,12]$. Despite the paucity of quality data on TPE in pediatric anti-NMDA receptor encephalitis, one review disclosed a trend toward better outcomes when TPE was administered early and when used in combination with steroids [13]. Although corticosteroids and IVIG are more common therapies for anti-NMDA receptor encephalitis than TPE is [3], in severe refractory autoimmune encephalitis, TPE has shown improvement that lasts for several months in some patients when used in combination with other treatments or as the only treatment [14]. In a case report, the use of corticosteroids (methylprednisolone) and IVIG failed to produce any improvement in one patient's severe clinical conditions, which improved following the third course of TPE [15].

In this study, there were few adverse events associated with the use of TPE; only once did hypotension and twice did clots in the TPE tubes lead to interruptions in TPE procedures. No serious adverse events or treatment-related deaths were detected. Hypotension occurred during 30 (25.4\%) procedures, which was higher than the previously reported occurrence of hypotension during TPE for antiNMDA receptor encephalitis (11\%) [10]. The difference 


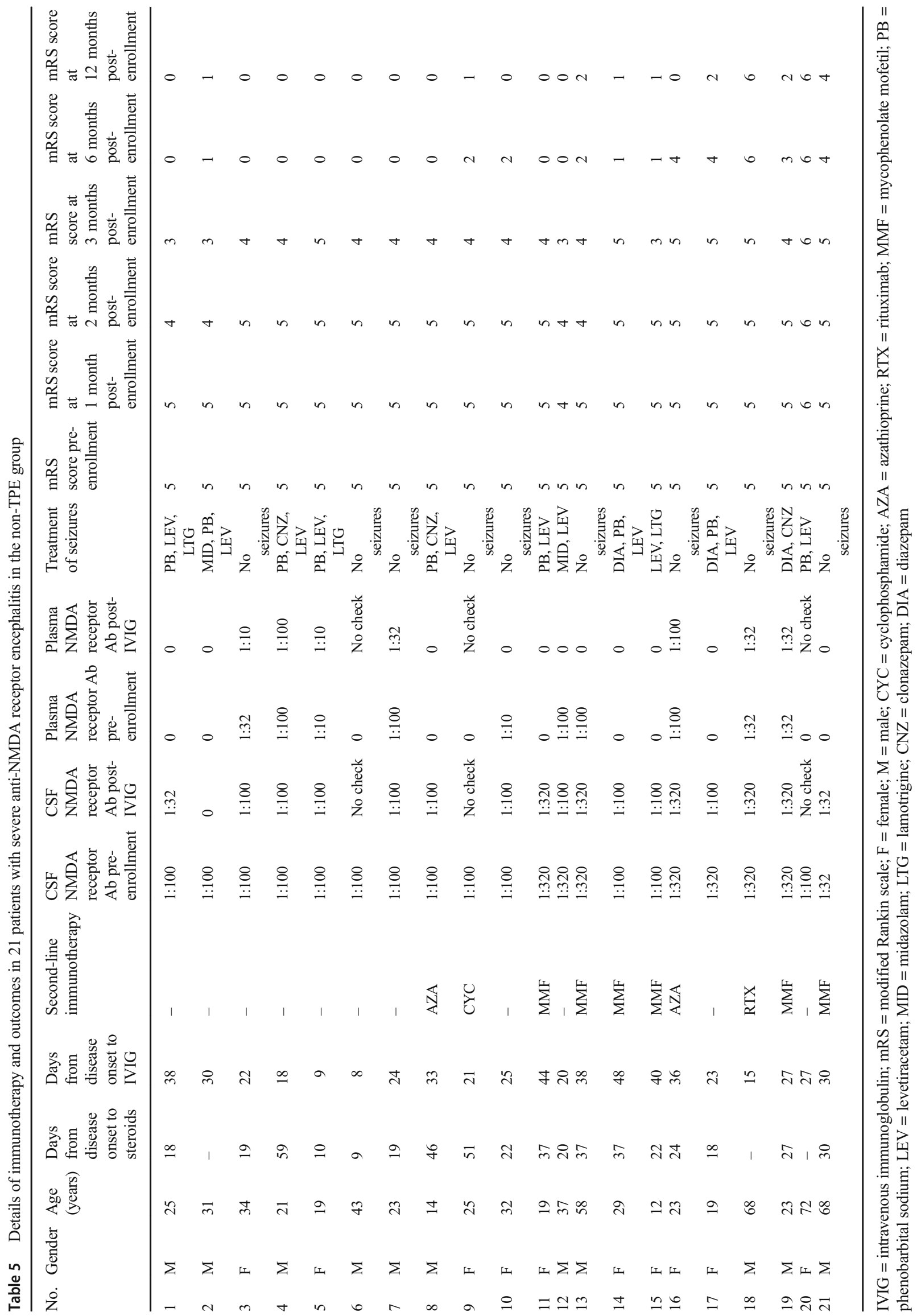


may be because in this study, there were more patients with severe disease who had autonomic dysfunction, such as hypotension, prior to TPE. A previous Swedish study [16] including 20,485 TPE procedures reported that hypotension occurred 186 times, of which 29 instances were transient hypotension, 108 required symptomatic treatments, and 49 resulted in discontinuation of TPE. Thus, hypotension is a common adverse reaction associated with TPE procedures. Although hypotension in most patients resolves after treatment, greater attention should still be paid to this condition, especially in anti-NMDA receptor encephalitis patients with autonomic dysfunction. At the same time, we should also pay attention to the prevention of coagulation in TPE tubes during TPE. Although tube coagulation does not harm the patient directly, it may lead to the discontinuation of TPE. Therefore, anticoagulants should be properly used and adjusted.

The increase in existing involuntary movements was a unique adverse event during the TPE procedures in patients with anti-NMDA receptor encephalitis, likely due to the filtration of medications routinely used to control the disease symptoms. In this study, involuntary movements increased during 43 (36.4\%) procedures; during 42 of these 43 procedures, patients needed sedatives or increased doses of the original sedatives to continue TPE. This phenomenon has not been reported during TPE used for the treatment of other nervous system diseases $[9,16,17]$. The main reason is that there are more prominent involuntary movements in patients with antiNMDA receptor encephalitis; for example, all 19 patients had involuntary movements prior to receiving TPE in this study. In the clinic, continuous-infusion sedatives and neuromuscular blocking agents are administered to patients with severe anti-NMDA receptor encephalitis, with the aim of limiting abnormal movements $[18,19]$. The amounts of medications, such as continuous-infusion midazolam or propofol, which we routinely used to control the symptoms of involuntary movements, were probably filtered during TPE procedures, resulting in significantly decreased concentrations of the medications in the blood and thus increased involuntary movements. For this reason, the proper addition of sedatives and/or muscle relaxants during TPE should be used in patients with obvious involuntary movements.

The preference of specific treatment options for patients with anti-NMDA receptor encephalitis remains a matter of debate, and numerous authors have reported conflicting data on the subject, particularly with regard to the role of TPE, which in the opinion of Dalmau et al. [20] should not be used routinely. First, this opinion could potentially be because this technique is generally considered more invasive and, therefore, may be reserved for particularly severe cases. Second, it is worth noting that the efficacy of TPE may be limited for patients with this disease, considering that removal of circulating antibodies may work systemically but does not alter the pathological process inside the central nervous system, in which abundant intrathecal production of autoantibodies occurs and infiltrates of plasma cells and plasmablasts have been previously described [21,22].

Among the patients with available information, our data suggest rapid efficacy in patients in whom first-line immunotherapy consisted of a combination of TPE with steroids and IVIG, possibly supporting a role of the combination of immunotherapies with peripheral and central actions. The NMDA receptor antibody titers in the CSF and/or plasma decreased or were negative after TPE in $94.7 \%$ of patients. The potential role for antibody removal via TPE in patients with anti-NMDA receptor encephalitis is supported by the direct pathogenic role of anti-NMDA receptor antibodies [5]. However, because the production of antibodies occurs intrathecally in patients with this disease [21], the removal of antibodies from the peripheral circulation via TPE may, in fact, alter the peripheral immunological response and possibly reduce the trafficking of antibodies and lymphocytes to the central nervous system; therefore, TPE should also be performed in combination with other therapies that modify intrathecal immune-mediated disease, such as steroids. IgG antibodies need to equilibrate between the intravascular and extravascular spaces. Moreover, in anti-NMDA receptor encephalitis, antibodies also need to equilibrate between the plasma and CSF. High initial antibody titers in the CSF are associated with poorer neurological outcomes and longer response times to treatment. Prior guidelines have indicated that 5-6 TPE procedures on alternating days are necessary to allow time for antibodies to equilibrate between the plasma and CSF [4].

TPE and immunoadsorption (IA) constitute two possibilities to eliminate pathogenic antibodies from patients' plasma. Previous studies have suggested that the addition of IA to immunosuppression therapy may accelerate recovery in autoimmune encephalitis, particularly in patients with antibodies targeting neuronal surfaces [23, 24]. To compare the efficacy and safety of TPE and IA, a study investigated the disease courses and treatment effects of 21 patients with autoimmune encephalitis who were randomly assigned to receive TPE $(n=11)$ or IA $(n=10)$. The results showed that both TPE and IA resulted in moderate to marked clinical improvement, with low rates of adverse events [25]. Even if patients did not respond to the TPE, none of them worsened in either this study or the study from Heine $\mathrm{J}$ et al. [25]. However, in this study, the TPE group exhibited $31.6 \%$ and $63.2 \%$ clinical improvement after 1 month and 2 months, respectively, which was not as successful as the finding that 
TPE resulted in moderate to marked clinical improvement $(67 \%)$ in the prior study [25]. The reasons for this difference are as follows. First, patients in the current study had more severe conditions than those in Heine J et al.'s study [25]. Patients with an mRS score of 5, respiratory failure requiring mechanical ventilation, disturbances of consciousness, or status epilepticus were enrolled in this study. Only 7 of the 21 patients were considered severe patients who were admitted to the ICU in Heine J et al.'s study [25]. Second, patients in the current study were refractory, and none experienced improvements after steroids and/or IVIG treatment for at least 10 days, whereas 7 patients did not accept either high-dose cortisone or IVIG before treatment with TPE or IA in Heine J et al.'s study [25].

There were several limitations in the current study. First, it included patients from a single center, and the sample size was relatively small. Nonetheless, this pilot study did provide useful information, as the TPE treatment was relatively safe and feasible for severe refractory anti-NMDA receptor encephalitis. However, it is necessary to emphasize the possible adverse events that may occur during TPE. Second, systematic comparisons between the three first-line modalities (corticosteroids, IVIG, and TPE) are unavailable, and the exact order of the treatments was not defined in our study. Early TPE combined with medications might control the disease faster and lead to a shorter disease duration and better prognosis for patients with severe conditions than treatment with medications alone. Given the importance of time-sensitive treatment, further studies may illuminate the ideal first-line treatment for severe anti-NMDA receptor encephalitis.

In summary, combined TPE immunotherapy is not harmful in terms of long-term outcome, and there may be a faster benefit in the very short term for patients with severe refractory anti-NMDA receptor encephalitis. TPE may in turn reduce hospitalization time, drug needs, and possibly short-term morbidity and should therefore be considered as an available treatment. TPE may even be considered for earlier use, but additional studies are required to determine the best treatment strategy for critically ill patients with progressive and lifethreatening anti-NMDA receptor encephalitis.

Acknowledgements This project was supported by the Chinese Natural Science Fund No. 81671037 (to Yingying Su). We thank Dr. Hongzhi Guan, Dr. Haitao Ren, and Dr. Yanhuan Zhao from the Department of Neurology, Peking Union Medical College Hospital, Chinese Academy of Medical Sciences, China, for performing anti-NMDA receptor antibody assays and for technical support.

\section{Compliance with Ethical Standards}

This study was approved by the Ethics Committee of Xuanwu Hospital, Capital Medical University, adhered to the tenets of the Declaration of Helsinki.
Conflict of Interest The authors declare that they have no conflict of interest.

Required Author Forms Disclosure forms provided by the authors are available with the online version of this article.

\section{References}

1. Dalmau J, Graus F (2018) Antibody-Mediated Encephalitis. N Engl J Med 378(9):840-851.

2. Dalmau J, Tuzun E, Wu HY, et al. (2007) Paraneoplastic anti-Nmethyl-D-aspartate receptor encephalitis associated with ovarian teratoma. Ann Neurol 61(1):25-36.

3. Titulaer MJ, McCracken L, Gabilondo I, et al. (2013) Treatment and prognostic factors for long-term outcome in patients with antiNMDA receptor encephalitis: an observational cohort study. Lancet Neurol 12(2):157-165.

4. Schwartz J, Padmanabhan A, Aqui N, et al. (2016) Guidelines on the Use of Therapeutic Apheresis in Clinical Practice-EvidenceBased Approach from the Writing Committee of the American Society for Apheresis: The Seventh Special Issue. J Clin Apher 31(3):149-162.

5. Moscato EH, Peng X, Jain A, et al. (2014) Acute mechanisms underlying antibody effects in anti-N-methyl-D-aspartate receptor encephalitis. Ann Neurol 76(1):108-119.

6. DeSena AD, Noland DK, Matevosyan K, et al. (2015) Intravenous methylprednisolone versus therapeutic plasma exchange for treatment of anti-n-methyl-d-aspartate receptor antibody encephalitis: A retrospective review. J Clin Apher 30(4): 212-216.

7. Graus F, Titulaer MJ, Balu R, et al. (2016) A clinical approach to diagnosis of autoimmune encephalitis. Lancet Neurol 15(4):391404.

8. Sulter G, Steen C, De Keyser J. (1999) Use of the Barthel index and modified Rankin scale in acute stroke trials. Stroke 30(8):1538 1541.

9. Ebadi H, Barth D, Bril V (2013) Safety of plasma exchange therapy in patients with myasthenia gravis. Muscle Nerve 47(4):510-514.

10. Pham HP, Daniel-Johnson JA, Stotler BA, et al. (2011) Therapeutic plasma exchange for the treatment of anti-NMDA receptor encephalitis. J Clin Apher 26(6):320-325.

11. Smith JH, Dhamija R, Moseley BD, et al. (2011) N-methyl-Daspartate receptor autoimmune encephalitis presenting with opsoclonus-myoclonus: treatment response to plasmapheresis. Arch Neurol 68(8):1069-1072.

12. Shahani L (2015) Steroid unresponsive anti-NMDA receptor encephalitis during pregnancy successfully treated with plasmapheresis. BMJ Case Rep 2015;2015.

13. Suppiej A, Nosadini M, Zuliani L, et al. (2016) Plasma exchange in pediatric anti-NMDAR encephalitis: A systematic review. Brain Dev 38(7):613-622.

14. Jaben EA, Winters JL. (2012) Plasma exchange as a therapeutic option in patients with neurologic symptoms due to antibodies to voltage-gated potassium channels: a report of five cases and review of the literature. J Clin Apher 27(5):267-273.

15. Rypulak E, Borys M, Piwowarczyk P, et al. (2016) Successful treatment of anti-NMDA receptor encephalitis with a prompt ovarian tumour removal and prolonged course of plasmapheresis: A case report. Mol Clin Oncol 5(6):845-849.

16. Norda R, Stegmayr BG (2003) Therapeutic apheresis in Sweden: update of epidemiology and adverse events. Transfus Apher Sci 29(2):159-166. 
17. Kim SH, Kim W, Huh SY, et al. (2013) Clinical efficacy of plasmapheresis in patients with neuromyelitis optica spectrum disorder and effects on circulating anti-aquaporin-4 antibody levels. J Clin Neurol 9(1):36-42.

18. Chen X, Li JM, Liu F, et al. (2016) Anti-N-methyl-D-aspartate receptor encephalitis: a common cause of encephalitis in the intensive care unit. Neurol Sci 37(12):1993-1998.

19. Howard CM, Kass JS, Bandi VD, et al. (2014) Challenges in providing critical care for patients with anti-N-methyl-D-aspartate receptor encephalitis. Chest 145(5):1143-1147.

20. Dalmau J, Lancaster E, Martinez-Hernandez E, et al. (2011) Clinical experience and laboratory investigations in patients with anti-NMDAR encephalitis. Lancet Neurol 10(1):63-74.

21. Martinez-Hernandez E, Horvath J, Shiloh-Malawsky Y, et al. (2011) Analysis of complement and plasma cells in the brain of patients with anti-NMDAR encephalitis. Neurology 77(6):589593.
22. Bartolini L, Muscal E (2017) Differences in treatment of antiNMDA receptor encephalitis: results of a worldwide survey. $\mathrm{J}$ Neurol 264(4):647-653.

23. Dogan OM, Golombeck KS, Bien C, et al. (2016) Immunoadsorption therapy in autoimmune encephalitides. Neurol Neuroimmunol Neuroinflamm 3(2):e207.

24. Kohler W, Ehrlich S, Dohmen C, et al. (2015) Tryptophan immunoadsorption for the treatment of autoimmune encephalitis. Eur J Neurol 22(1):203-206.

25. Heine J, Ly LT, Lieker I, et al. (2016) Immunoadsorption or plasma exchange in the treatment of autoimmune encephalitis: a pilot study. J Neurol 263(12):2395-2402.

Publisher's Note Springer Nature remains neutral with regard to jurisdictional claims in published maps and institutional affiliations. 